\title{
Gerontology
}

\section{A Two-Phase Pathogenetic Model of Depression after Stroke}

\author{
Katja Werheid \\ Department of Psychology, Humboldt Universität zu Berlin, Berlin, Germany
}

\section{Key Words}

Depression · Poststroke depression · Stroke

\begin{abstract}
Depression is among the major long-term complications of cerebral stroke. Occurring in about $30 \%$ of all stroke survivors, 'poststroke depression' (PSD) is known to be associated with prolonged recovery, reduced quality of life, and increased mortality. Research over the past 25 years has enlarged our knowledge about organic and psychosocial risk factors, but their interaction is still unclear. In this paper, we start by reviewing and discussing pathogenetic PSD models that were proposed in the 1980s and 1990s. Based on these earlier approaches and on longitudinal research published since that time, a biphasic model is proposed that reflects the current knowledge on the emergence of PSD. The model integrates premorbid, stroke-related and psychosocial risk factors contributing to the emergence of PSD within the first 2 years after stroke. The distinction between 'reactive' and 'organic' PSD has been dropped, and groups of well-documented predictors are recommended for future research. Finally, we will outline possible implications of the model for prevention and therapy.
\end{abstract}

(c) 2015 S. Karger AG, Basel

\section{KARGER}

(C) 2015 S. Karger AG, Basel

0304-324X/15/0621-0033\$39.50/0

E-Mail karger@karger.com

www.karger.com/ger

\section{Introduction}

Depression is among the most frequent mental disorder after stroke, with an estimated prevalence of 30\% [1]. Due to its high frequency and a presumed specific etiology, a proper term has been coined for the phenomenon: poststroke depression (PSD). Counted among the major malign long-term sequelae of stroke, PSD is associated with prolonged recovery [2] and an increased risk of recurrent and fatal stroke [3].

The frequency of PSD increases in lockstep with stroke prevalence. Due to demographic changes, the number of stroke events in Europe is projected to increase from 1.1 million per year in 2000 to 1.5 million in 2025 [4]. Thanks to advances in emergency medicine and acute care, the number of stroke survivors has consistently increased over the past 3 decades [5]. Currently, two thirds of all cases survive stroke, yet half of these survivors suffer from persisting impairment [6]. In one third of the survivors, quality of life is additionally reduced by a depressive disorder [7].

Despite these facts, our knowledge about PSD is still fragmentary. Research is complicated by long periods of incidence amounting up to 2 years after stroke, by intermittent exacerbation of PSD, and by changes of care providers when proceeding from acute to stationary or ambulant care [8]. However, these obstacles are not unique to PSD, but shared by many psychiatric complications of chronic or life-threatening diseases, e.g. in multiple sclero- 
Fig. 1. Schematic illustration of existing dualistic models of PSD pathogenesis.

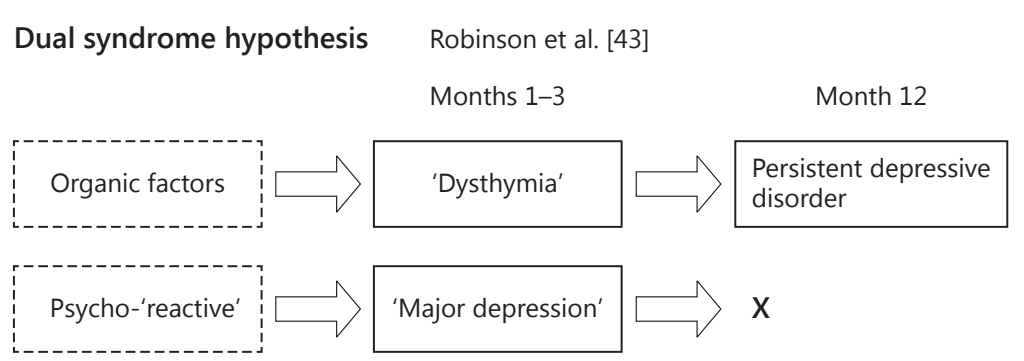

Conflicting evidence e.g. Gainotti et al. [11, 12]

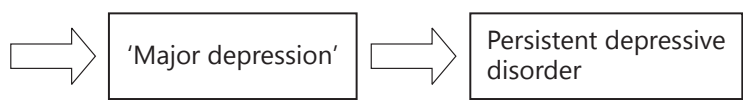

sis or cancer, and should not prevent us from developing procedures for early identification and effective treatment. A pathogenetic model involving the whole period of incidence is the starting point for this endeavor. The present paper will review pathogenetic models proposed in the 1980s and 1990s. Based on these earlier approaches and on longitudinal research published since that time, a twophase model is proposed that reflects the current knowledge about the emergence of PSD. Finally, possible implications of the model are outlined for research and practice.

\section{Dualistic Models of the PSD Pathogenesis}

Early concepts of PSD were oriented towards a dualism of organic and 'endogenous' psychiatric disease (fig. 1). Based on the findings of their pioneering longitudinal study, the Baltimore research group postulated two different syndromes $[9,10]$. A mild depressive syndrome similar to the DSM-IV diagnosis 'minor depression' was thought to be related to organic factors and to persist as a chronic 'poststroke depression' condition after 2 years. In contrast, a more severe initial syndrome similar to 'major depression', associated with lesions in the left frontal cortex and underlying basal ganglia, was thought to be temporary or 'reactive' and to disappear within 2 years after stroke. This dual syndrome hypothesis was challenged by later prospective longitudinal research, revealing a considerable number of cases with persisting major depression or short-lived minor depression syndromes as well as later onset of major depression [11, 12].
Later concepts of PSD rather focused on the sequential evolvement of PSD in different critical periods within 2 years after stroke [13]. Herrmann and Wallesch [14] related these stages to the three typical rehabilitation phases: acute, postacute, and chronic phase (after discharge). They proposed that in the acute phase after stroke, PSD would be related to primary factors, such as premorbid disposition, lesion site, and configuration. Later on, during postacute rehabilitation, when the patients realize that their impairments may be long-lasting or even permanent, secondary psychological factors, such as coping style, would gain in importance. After discharge from the rehabilitation center, reintegration into the social environment, involving a change of roles from being a patient to being 'disabled', was proposed as the tertiary factor that might trigger depression. Despite the distinction of three phases, the dualism of 'organic' and 'psychosocial' factors was kept by the model, as the authors proposed a progression from organic to psychosocial risk factors that could trigger PSD.

Gainotti et al. [12] tested the central predictions of both models in their longitudinal study sample. First, they found that the symptom profiles of patients classified as having PSD were very similar at 2, 4 , and 6 months after stroke, but differed from a control sample with major depression without stroke. In PSD patients, symptoms of anxiety, catastrophic reactions, hyperemotionalism, and diurnal mood variations were more frequent, whereas in the 'nonorganic' major depression sample, depressed mood, guilt feelings, and suicidal thoughts were more frequent. This finding argued against the two-syndrome hy- 


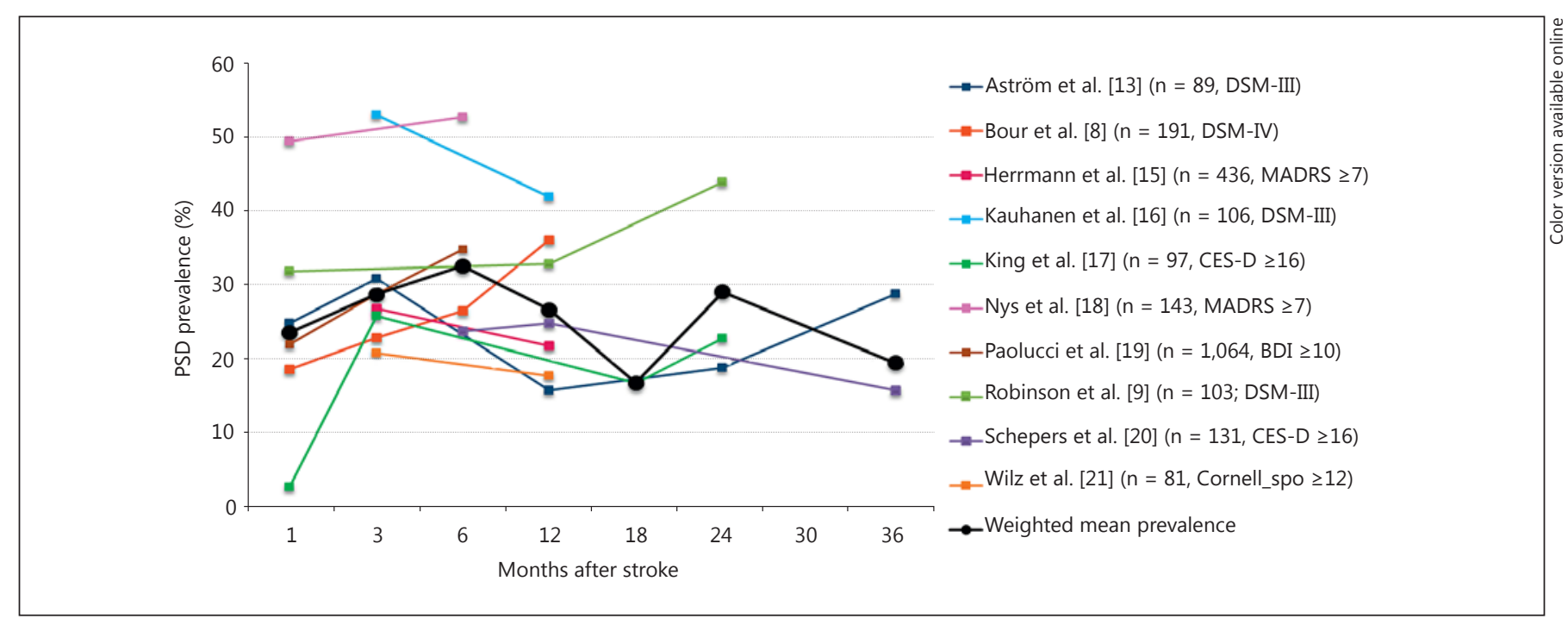

Fig. 2. Overview of PSD rates in 11 prospective longitudinal studies of stroke survivors ( $\mathrm{n}>80$, at least 2 assessments) from 1987 to 2013, with a total sample size of 2,641 patients. The thick black line represents mean depression rates weighted according to the sam- ple size. MADRS = Montgomery-Åsberg Depression Rating Scale; CES-D = Center for Epidemiological Studies Depression Scale; BDI = Beck Depression Inventory; Cornell_spo = Cornell Scale, filled in by spouses. pothesis. As a second finding, Gainotti et al. [12] found no significant relationship between lesion location, presence of PSD, and time elapsed since stroke, which was not in line with the assumptions of the model of Herrmann and Wallesch [14].

\section{Review of Prospective Longitudinal Research}

Our review of the literature involved prospective longitudinal studies available in the MEDLINE and PUBMED databases between 1987, the year in which the first longitudinal data on PSD by Robinson et al. [9] appeared, and January 1, 2014. Key words were 'stroke' AND 'depress' AND 'prospective longitudinal'. In case of multiple papers on the same project, only the main publication was selected for each study. The references of each study were checked for citations of further studies in this field. Studies were selected if they fulfilled the following criteria: (a) an initial sample of at least 80 stroke patients included in the study, (b) $\geq 2$ assessments of depression within the first 2 years after stroke, and (c) reporting the rates of stroke patients that fulfilled clinical consensus criteria of depression (ICD-10; DSM-IV) or ranged above a commonly accepted cutoff in a depression questionnaire. Studies that reported group means in depression questionnaires without distinguishing between subclinical symptoms and depressive disorder were not included. Ten prospective longitudinal studies fulfilled these criteria for PSD $[8,9,13,15-21]$. The rates of patients classified as depressive were pooled and averaged across studies, so that the resulting mean rates were weighted according to the sample size. A total of 2,641 patients were considered in this review. The resulting mean of depression rates showed a biphasic pattern, with a rise in depressive symptoms within the first 6 months after stroke, which drops slightly around 12 months, and rises again within the second year after stroke (fig. 2).

It should be noted that our overview of studies is exploratory and limited in several aspects. First, most of the considered studies are hospital- or rehabilitation-based rather than community-based. Thus, they may be selective, as not all stroke patients may be treated in institutions, and as depression may be assessed relatively late in the course of the disease [22]. As a further aspect of selectivity, which is very common in studies on PSD, patients with aphasia might be underrepresented, as the questionnaires used here were all based on verbal reports.

Our review is further limited by large methodological variations between the studies. Although we attempted to control for different sample sizes by weighting the mean PSD rates, the employed diagnostic procedures also varied largely. In addition, the initial sample sizes were not overly large, i.e. around 100 in most of the studies, and 
further diminished in the course of the study due to high comorbidity, mortality, and a shift of living conditions in stroke patients, resulting in dropout rates of around 30\% per year. Moreover, only one of the reviewed studies [17] reported depression rates at 18 months after stroke. Thus, the decrease in prevalence rates from nearly $30 \%$ at 12 months to $<20 \%$ at 18 months relies on restricted data. However, even if disregarding the 18 months' data point, there is a slight decline in prevalence rates between 6 and 12 months based on multiple large studies. However, this trend does not continue to decrease until 24 months after stroke, but is, even without the 18 months' data point, compatible with the view that a second cluster of risk factors may increase prevalence rates during the second year after stroke. Despite these limitations, it is notable that the overall pattern emerging from these studies is biphasic, with a second rise in the second year after stroke, which may reflect problems of adjustment as proposed by Herrmann and Wallesch [14].

\section{Risk Factors for PSD}

To explore the pathogenesis of PSD, a large number of potential risk factors have been examined over the past 30 years. Demographic, stroke-related, premorbid, psychosocial, and neurobiological factors were investigated for associations with PSD. Reviewing the existing literature, Hackett and Anderson [1] counted no less than 87 investigated predictors. They identified 4 major variables that had been investigated in at least 5 studies and consistently proved to be predictors of later depression: physical disability, stroke severity, cognitive impairment, and psychosocial factors, such as social support after stroke. Evidence regarding demographic variables, such as older age and female gender, was divided at that point in time.

Regarding the pathogenetic models of PSD, Hackett and Anderson [1] found that most studies provided rather explanatory than predictive models. Concluding that no reliable data were available to allow an easy identification of patients with depression after stroke, they emphasized the importance of predictive models, which permit to estimate the risk of later depression on the basis of acute or postacute variables. Finally, they recommended additional research in this area, 'not only in terms of increasing our understanding of etiological risk factors for depression in the setting of stroke but also in advancing health care delivery to improve stroke rehabilitation outcomes' [1].

Since then, several longitudinal studies have been published, such as the large Italian DESTRO study [23], reac- tualizing the importance of the female gender, the history of depression, and the history of stroke as risk factors, and the Sydney Study [24], emphasizing the importance of apathy. Also, as an interesting and rarely considered factor, Storor and Byrne [25] revealed that premorbid personality affected the risk of PSD.

\section{Demographic Risk Factors}

The impact of demographic risk factors on PSD is controversial and may be less pronounced than in other mental diseases. The female gender, although examined in most studies, was sometimes identified as a risk factor, but rather than being clearly related to PSD, it was linked to depressive disorders in general. Age $<65$ years was by some, but not all, studies identified as a predictor of PSD, a finding which was explained by differences in the occupational status, as younger people's lives might be more affected than that of retired patients [10]. This explanation, together with the varying pattern found in the literature, suggests that demographic factors might not act as independent predictors, but as mediators for other risk factors [9].

\section{Premorbid Risk Factors}

Several premorbid health conditions, such as a history of depressive disorder as well as a history of stroke or cardiovascular disease, are well-established risk factors for PSD [26]. Others, such as personality and coping skills [27] or premorbid neurobiological risk factors, are often recommended for future research [28], but are not yet fully understood.

\section{Stroke-Related Risk Factors}

Stroke-related factors include all factors directly related to cerebral damage caused by the ischemic or hemorrhagic event. There is no evidence of a significant influence of the stroke type. Location of the lesion in terms of the hemisphere in which the stroke occurred has been controversially discussed. While initial research on PSD reported evidence of a higher PSD risk in left-hemisphere stroke [9], this has not been supported by the majority of studies according to more recent large-scale reviews [1]. It has been proposed that specific lesions such as the disruption of frontostriatal circuits may be associated with PSD [29]. To date, evidence for more specific hypotheses on lesion localization has been limited by methodological diversity across studies [22].

Under the topic of 'stroke-related risk factors', many studies also subsume the degree of impairment, often estimated by impairments of activities of daily living, as well
Werheid 
Fig. 3. Proposed two-phase pathogenetic model of depression after stroke (PSD).

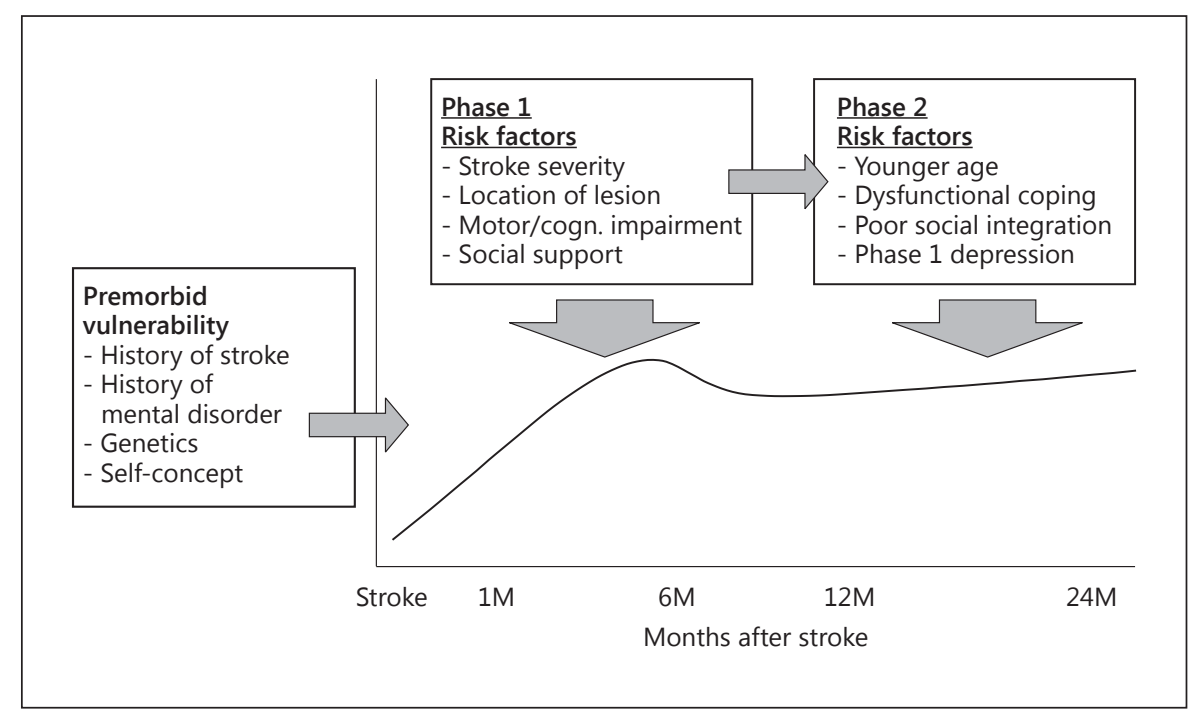

as cognitive impairment, language impairment, or neglect. Although these risk factors have often been addressed, their relationship with depression is far from definite [30], which suggests the presence of mediators and moderators.

\section{Psychosocial Risk Factors}

Several psychosocial risk factors, such as the amount of social support perceived by the patient, might affect the level of depressive symptoms already in early phases after stroke [31]. Nevertheless, these factors might also predict PSD in later time periods, when reintegration into everyday life is relevant $[26,32]$.

\section{A Two-Phase Model of PSD Pathogenesis}

Integrating the sequential evolvement hypothesis and the current knowledge about risk factors, we propose a two-phase model of PSD pathogenesis. As displayed in figure 3, the model distinguishes between an early and a late period, in which depressive disorders likely emerge. In accordance with recent studies analyzing longitudinal data with hierarchical regression methods, the model postulates partly dissociable sets of risk factors for earlyand late-onset depressiveness. However, these factors are not grouped in a dualistic way. While the influence of stroke-related factors is not restricted to the first months after stroke, psychosocial factors may exert their influence not only in later phases $[21,33]$ but also within the first weeks after stroke [31].

A Two-Phase Pathogenetic Model of Depression after Stroke
Regarding phase 1, early-onset depressiveness in the first weeks after stroke is, according to the literature, associated with stroke-related factors, such as functional impairments due to motor or language deficits. Our model additionally emphasizes the role of premorbid factors. Especially neurobiological factors, such as a preactivated stress system, and psychological premorbid factors, such as self-concept or self-efficacy beliefs, have often been proposed $[26,32,34]$ but rarely considered in large longitudinal studies. Notably, early-onset depressiveness itself represents an important predictor of later, persisting depressive disorders [35].

In phase 2 , about 1 year after stroke, most patients have finished rehabilitation programs, but are typically still involved in a long-term reintegration process, and are probably confronted with their persisting impairments. Activity of daily living impairment is still a major determinant of depression in this late phase. However, the relationship with depression is seemingly not unitary, but mediated by coping skills and the degree of social integration [25]. Patients who experience their daily lives as stressful and find it difficult to adapt to their environment are at a higher risk of depression [36].

Although our model as a whole is in line with the current knowledge on PSD risk factors, it does not reflect the findings of every study in the field. One obvious reason may be the large variation of the study samples, ranging from slightly impaired clients of outpatient wards to severely impaired patients in rehabilitation clinics. A further source of variance is the above-mentioned diversity of syndromes and impairments following stroke. Despite 
these limitations, it is remarkable that the above-reported pattern emerging from the literature is complemented by the clinicians' observation of a late 'second rise' of depression [17], which seems less closely related to stroke severity than early-onset depressiveness [37].

\section{Implications of the Model}

The primary aim of our two-phase model was to integrate the existing knowledge on the PSD risk factors in order to stimulate further longitudinal research and to provide a basis for the development of preventive and therapeutic strategies. In the following section, we will outline the most important implications for research and practice. First, with regard to prospective longitudinal research in general, there is a need for more studies involving large consecutive hospital-based sampling. Pure register-based studies may have been valuable in the past, but as the main groups of risk factors are well confirmed now, the time is ripe to generate hypothesis-driven designs with consecutive assessments. These assessments should include the supposed 'intermediate' phase between 6 and 18 months and long-term follow-ups of at least 2 years. The change of health care providers and the high rate of dropouts is a serious problem and should be combatted with any means, especially those developed in successful epidemiological research.

Second, as formulated by a recent review on the predictors of depression after stroke [21], the proposed sets of risk factors should be validated by regression-based statistical methodology [33]. These methods require large longitudinal data sets, but with refined methodology, the stroke- and age-typical variability is not limited by multiple selection criteria to the same extent [38], but can be statistically controlled by carefully selected and standardized descriptive parameters, such as scales measuring initial stroke severity. Third, parameters of premorbid neurobiological and self-concept-related risk factors as well as indicators of patient-environmental fit, often proposed as promising candidates, should be further explored. Together with these research activities, our model may contribute to proceed from accumulating findings on relevant risk factors towards the identification of time-bound sets of risk factors, which may interact with each other. Specific combinations of risk factors may be linked to PSD exacerbation in different time windows.

Knowledge of these predictor sets may allow for better targeting of interventions for preventing, treating and managing depression after stroke. For example, a past history of depression, one of the well-confirmed premorbid factors in the model, should be part of the routine assessment in acute neurology, in the same way as a history of prior strokes or transitory ischemic attacks. Another premorbid factor, i.e. self-concept and coping strategies, suffers from heterogeneity and limited availability of economical standardized assessment tools. Such measures should be developed. Their results, for example a preference for a certain way of coping, can be documented in the reports handed over to postacute or ambulant treatment.

In phase 1, further preventive strategies should be established. First, as depressive symptoms have proven to be predictive of later depressive disorders [39], these depressive symptoms should be assessed and documented. Special attention should be given to the assessment of aphasic patients in this context [40]. The finding that the self-perceived degree of social support and satisfaction with care seems to already be crucial in this phase [41] should be incorporated in the education of health professionals and in the counseling of caregivers and relatives. Also, there is an urgent need for intervention studies with pharmacological or nonpharmacological agents. Notably, although in depression without stroke it is the combination of both strategies that has proven most promising, there are no studies on combination therapy in PSD. The recent increased prescription of selective serotonin reuptake inhibitors in stroke is based on a study indicating better rehabilitation results in medicated stroke patients [42], but the mechanisms of this effect are not yet understood, and unwarranted bi-effects of antidepressants in nondepressive stroke patients are underexplored.

Finally, in phase 2 ambulant therapy, integration is the central topic. More studies including at least 2-year follow-ups should be conducted. Similar to research on resilience, predictors of successful reintegration and successful prevention of PSD should be explored, in addition to examining those patients suffering from PSD.

\section{Acknowledgements}

Many thanks to Inga-Marlen Pontow for assisting in the literature review and for valuable discussions, and to Yasmina Giebeler for assistance in the literature research and manuscript preparation.
Werheid 


\section{References}

1 Hackett ML, Anderson CS: Predictors of depression after stroke: a systematic review of observational studies. Stroke 2005;36:22962301.

2 Robinson RG, Spalletta G: Poststroke depression: a review. Can J Psychiatry 2010;55:341349.

3 Pan A, Sun Q, Okereke OI, Rexrode KM, Hu FB: Depression and risk of stroke morbidity and mortality: a meta-analysis and systematic review. JAMA 2011;306:1241-1249.

4 Truelsen T, Piechowski-Jozwiak B, Bonita R, Mathers C, Bogousslavsky J, Boysen G: Stroke incidence and prevalence in Europe: a review of available data. Eur J Neurol 2006; 13:581598.

5 Kunst AE, Amiri M, Janssen F: The decline in stroke mortality exploration of future trends in 7 Western European countries. Stroke 2011;42:2126-2130.

6 Roger VL, Go AS, Lloyd-Jones DM, Benjamin EJ, Berry JD, Borden WB, et al: Executive summary: heart disease and stroke statistics - 2012 update: a report from the American Heart Association. Circulation 2012;125: 188-197.

7 Carod-Artal FJ, Egido JA: Quality of life after stroke: the importance of a good recovery. Cerebrovasc Dis 2009;27:204-214.

8 Bour A, Rasquin S, Aben I, Boreas A, Limburg M, Verhey F: A one-year follow-up study into the course of depression after stroke. J Nutr Health Aging 2010;14:488-493.

9 Robinson RG, Bolduc PL, Price TR: Two-year longitudinal study of poststroke mood disorders: diagnosis and outcome at one and two years. Stroke 1987;18:837-843.

10 Robinson RG, Starr LB, Kubos KL, Price TR: A 2-year longitudinal study of post-stroke mood disorders. Findings during the initial evaluation. Stroke 1983;14:736-741.

11 Gainotti G, Azzoni A, Gasparini F, Marra C, Razzano C: Relation of lesion location to verbal and nonverbal mood measures in stroke patients. Stroke 1997;28:2145-2149.

12 Gainotti G, Azzoni A, Razzano C, Lanzillotta M, Marra C, Gasparini F: The post-stroke depression rating scale: a test specifically devised to investigate affective disorders of stroke patients. J Clin Exp Neuropsychol 1997; 19:340-356.

13 Aström M, Adolfsson R, Asplund K: Major depression in stroke patients. A 3-year longitudinal-study. Stroke 1993;24:976-982.

14 Herrmann M, Wallesch CW: Depressive changes in stroke patients. Disabil Rehabil 1993;15:55-66.

15 Herrmann N, Black SE, Lawrence J, Szekely C, Szalai JP: The Sunnybrook Stroke Study: a prospective study of depressive symptoms and functional outcome. Stroke 1998;29:618624.
16 Kauhanen ML, Korpelainen JT, Hiltunen P, Brusin E, Mononen H, Maatta R, et al: Poststroke depression correlates with cognitive impairment and neurological deficits. Stroke 1999;30:1875-1880.

17 King RB, Shade-Zeldow Y, Carlson CE, Feldman JL, Philip M: Adaptation to stroke: a longitudinal study of depressive symptoms, physical health, and coping process. Top Stroke Rehabil 2002;9:46-66.

18 Nys GMS, van Zandvoort MJE, van der Worp HB, de Haan EHF, de Kort PLM, Jansen BPW, et al: Early cognitive impairment predicts longterm depressive symptoms and quality of life after stroke. J Neurol Sci 2006;247:149-156.

19 Paolucci S, Gandolfo C, Provinciali L, Torta $\mathrm{R}$, Toso V, Group DS: The Italian multicenter observational study on post-stroke depression (DESTRO). J Neurol 2006;253:556-562.

20 Schepers V, Post M, Visser-Meily A, van de Port I, Akhmouch M, Lindeman E: Prediction of depressive symptoms up to three years post-stroke. J Rehabil Med 2009;41:930-935.

21 Wilz G, Barskova T: Predictors of psychological and somatic components of poststroke depression: a longitudinal study. Top Stroke Rehabil 2007;14:25-40.

22 Bhogal SK, Teasell R, Foley N, Speechley M: Lesion location and poststroke depression: systematic review of the methodological limitations in the literature. Stroke 2004;35:794802.

23 Paolucci S, Gandolfo C, Provinciali L, Torta $\mathrm{R}$, Sommacal S, Toso V, et al: Quantification of the risk of poststroke depression: the Italian multicenter observational study DESTRO. Acta Psychiatr Scand 2005;112:272-278.

24 Brodaty H, Sachdev PS, Withall A, Altendorf A, Valenzuela MJ, Lorentz L: Frequency and clinical, neuropsychological and neuroimaging correlates of apathy following stroke - the Sydney Stroke Study. Psychol Med 2005;35: 1707-1716.

25 Storor DL, Byrne GJA: Pre-morbid personality and depression following stroke. Int Psychogeriatr 2006;18:457-469.

26 Ouimet MA, Primeau F, Cole MG: Psychosocial risk factors in poststroke depression: a systematic review. Can J Psychiatry 2001;46: 819-828.

27 Aben I, Denollet J, Lousberg R, Verhey F, Wojciechowski F, Honig A: Personality and vulnerability to depression in stroke patients: a 1-year prospective follow-up study. Stroke 2002;33:2391-2395.

28 Loubinoux I, Kronenberg G, Endres M, Schumann-Bard P, Freret T, Filipkowski RK, et al: Post-stroke depression: mechanisms, translation and therapy. J Cell Mol Med 2012; 16:1961-1969.

29 Vataja R, Leppavuori A, Pohjasvaara T, Mantyla R, Aronen HJ, Salonen O, et al: Poststroke depression and lesion location revisited. J Neuropsychiatry Clin Neurosci 2004; 16:156162.
30 Ramasubbu R, Robinson RG, Flint AJ, Kosier T, Price TR: Functional impairment associated with acute poststroke depression: the Stroke Data Bank study. J Neuropsychiatry Clin Neurosci 1998;10:26-33.

31 Lewin A, Jöbges M, Werheid K: The influence of self-efficacy and perceived social support on self-reported depressive symptoms during stroke rehabilitation. Neurolpsychol Rehabil 2013;23:546-562.

32 Whyte EM, Mulsant BH: Post stroke depression: epidemiology, pathophysiology, and biological treatment. Biol Psychiatry 2002;52: 253-264.

33 Shimoda K, Robinson RG: The relationship between poststroke depression and lesion location in long-term follow-up. Biol Psychiatry 1999;45:187-192.

34 Appelros P, Stegmayr B, Terent A: Sex differences in stroke epidemiology: a systematic review. Stroke 2009;40:1082-1090.

35 Verdelho A, Henon H, Lebert F, Pasquier F, Leys D: Depressive symptoms after stroke and relationship with dementia - a three-year follow-up study. Neurology 2004;62:905-911.

36 Robinson-Smith G, Johnston MV, Allen J: Self-care self-efficacy, quality of life, and depression after stroke. Arch Phys Med Rehabil 2000;81:460-464.

37 Folstein MF, Maiberger R, McGugh P: Mood disorder as a specific complication of stroke. J Neurol Neurosurg Psychiatry 1977;40: 1018-1020.

38 Werheid K, Koehncke Y, Ziegler M, Kurz A Latent change score modeling as a method for analysing the antidepressant effect of a psychosocial intervention in Alzheimer's disease. Psychother Psychosom 2015;84:159-166.

39 Lewin A, Volz M, Jöbges M, Werheid K: Predictivity of early depressive symptoms for post-stroke depression. J Nutr Health Aging, in press.

40 Kontou E, Thomas SA, Lincoln NB: Psychometric properties of a revised version of the Visual Analog Mood Scales. Clin Rehabil 2012;26:1133-1140.

41 Morrison V, Johnston M, Mac Walter R: Predictors of distress following an acute stroke: disability, control cognitions, and satisfaction with care. Psychol Health 2000;15:395-407.

42 Chollet F, Tardy J, Albucher JF: Fluoxetine for motor recovery after acute ischaemic stroke (FLAME): a randomised placebo-controlled trial. Lancet Neurol 2011;10:123-130.

43 Robinson RG, Lipsey JR, Rao K, Price TR: Two-year longitudinal study of poststroke mood disorders: comparison of acute-onset with delayed-onset depression. Am J Psychiatry $1986 ; 143: 1238-1244$.
A Two-Phase Pathogenetic Model of Depression after Stroke
Gerontology 2016;62:33-39 DOI: $10.1159 / 000381876$ 